\title{
Estimación de la carga de las enfermedades cardiovasculares atribuible a factores de riesgo modificables en Argentina
}

\author{
Adolfo Rubinstein, ${ }^{1,2}$ Lisandro Colantonio, ${ }^{1}$ Ariel Bardach, ${ }^{1,3}$ Joaquín Caporale, ${ }^{1,4}$ \\ Sebastián García Martí, ${ }^{1,2}$ Karin Kopitowski, ${ }^{2}$ Andrea Alcaraz, ${ }^{1}$ Luz Gibbons, ${ }^{1}$ \\ Federico Augustovski ${ }^{1,2}$ y Andrés Pichón-Rivière ${ }^{1}$
}

Forma de citar

Rubinstein A, Colantonio L, Bardach A, Caporale J, García Martí S, Kopitowski K, et al. Estimación de la carga de las enfermedades cardiovasculares atribuible a factores de riesgo modificables en Argentina. Rev Panam Salud Publica. 2010;27(4):237-45.

RESUMEN Objetivo. Estimar la carga de la enfermedad, su proporción atribuible a los principales factores de riesgo cardiovascular modificables y los costos médicos directos por hospitalización, asociados con las enfermedades coronarias y los accidentes cerebrovasculares en Argentina.

Métodos. Se elaboró un modelo analítico a partir de los datos de mortalidad en Argentina en 2005 y la prevalencia de los principales factores de riesgo cardiovascular (hipertensión arterial, hipercolesterolemia, sobrepeso, obesidad, hiperglucemia, tabaquismo actual y pasado, sedentarismo y consumo inadecuado de frutas y verduras). Se estimaron la carga de la enfermedad —años potenciales de vida perdidos (APVP) y años de vida saludable (AVISA) perdidosy los costos de hospitalización por las enfermedades cardiovasculares analizadas.

Resultados. En 2005 se perdieron en Argentina más de 600000 AVISA y se contabilizaron casi 400000 APVP por enfermedades coronarias y accidentes cerebrovasculares; $71,1 \%$ de los AVISA perdidos, 73,9\% de APVP y 76,0\% de los costos asociados son atribuibles a factores de riesgo modificables. La hipertensión arterial fue el factor de riesgo de mayor impacto, tanto en hombres como en mujeres: $37,3 \%$ del costo total, $37,5 \%$ de los APVP y $36,6 \%$ de los AVISA perdidos.

Conclusiones. La mayor parte de la carga de la enfermedad en Argentina por enfermedades cardiovasculares está relacionada con factores de riesgo modificables — por lo tanto evitables-y podría reducirse mediante intervenciones poblacionales y clinicas basadas en un enfoque de riesgo, que ya han demostrado ser efectivas en función del costo, asequibles y factibles en países como Argentina.

Palabras clave Enfermedades cardiovasculares; costo de enfermedad; años potenciales de vida perdidos; factores de riesgo; Argentina.

Instituto de Efectividad Clínica y Sanitaria, Buenos Aires, Argentina. La correspondencia se debe dirigir a Adolfo Rubinstein, Instituto de Efectividad Clínica y Sanitaria, Calle Viamonte No. 2149, piso 3, Capital Federal, C1056ABH, Buenos Aires, Argentina. Correos electrónicos: arubinstein@iecs. org.ar, adolfo.rubinstein@gmail.com

2 Servicio de Medicina Familiar y Comunitaria, Hospital Italiano de Buenos Aires, Buenos Aires, Argentina.

3 Programa de Prevención del Infarto en Argentina, Universidad Nacional de La Plata, Buenos Aires, Argentina.
En la mayoría de los países de ingresos bajos y medianos podría haber en las próximas décadas una acentuada aceleración en la tendencia de reducción de la tasa de mortalidad general que se viene observando en los últimos 30-40 años

\footnotetext{
4 Centro de Endocrinología Experimental y Aplicada, Universidad Nacional de La Plata, Buenos Aires, Argentina.
}

(1). Se ha informado que si bien a las enfermedades cardiovasculares, el cáncer, las enfermedades respiratorias crónicas y la diabetes corresponde alrededor de $60 \%$ de las muertes en el mundo, $80 \%$ de ellas ocurre en países de ingresos bajos y medianos. Se calcula que en 2015, cerca de 41 millones de personas morirán de enfermedades crónicas si no se conciertan acciones efectivas para su preven- 
ción y tratamiento. Casi la mitad de esas muertes correspondería a personas menores de 70 años en países en desarrollo, mientras que en los países de ingresos altos a estas enfermedades correspondería solamente $27 \%$ de las muertes en este mismo grupo de edad (2). A las enfermedades cardiovasculares, causa de alrededor de 18 millones de muertes anuales, corresponde $11 \%$ de la carga de la enfermedad en el mundo; esa carga tiene un crecimiento más acelerado en los países de ingresos medios y economías en transición (1).

En respuesta a esta epidemia, la Organización Mundial de la Salud (OMS) llamó a promover acciones eficaces a fin de reducir al menos en $2 \%$ anual la mortalidad por estas causas, lo que representaría 36 millones de muertes menos entre 2005 y 2015 (3). De hecho, se estima que la mortalidad por enfermedades coronarias y accidentes cerebrovasculares aumentará relativamente $145 \%$ en hombres y mujeres en los países en desarrollo entre 1990 y 2020, comparado con solo $28 \%$ en mujeres y $50 \%$ en hombres durante el mismo período en los países desarrollados (3).

Desde la perspectiva de la salud pública, las enfermedades cardiovasculares se deben prevenir mediante un enfoque de riesgo, es decir, con acciones dirigidas tanto a la población general como a las poblaciones en mayor riesgo de estas enfermedades y la implementación de intervenciones preventivas de eficacia demostrada. Datos científicos aportados por estudios epidemiológicos realizados en diferentes regiones han demostrado el efecto negativo de algunos factores de riesgo - como el tabaquismo, la diabetes mellitus, la hipertensión arterial, la dislipidemia, el sobrepeso y la obesidad, el sedentarismo y el bajo consumo de frutas y verduras - sobre la incidencia de las enfermedades cardiovasculares. Los resultados de la rama latinoamericana del estudio INTERHEART, que incluyó más de 3000 casos y controles, demuestran la validez general de esos factores de riesgo en esta parte del mundo (4).

En Argentina, la tasa de mortalidad por enfermedades cardiovasculares ajustada por la edad para hombres y mujeres en 2006 - incluidas las enfermedades coronarias y los accidentes cerebrovasculares- fue de 206,4 por 100000 habitantes (265,4 por 100000 hombres y 161,8 por 100000 mujeres), lo que representa $34,2 \%$ de las muertes y $12,6 \%$ de los años poten- ciales de vida perdidos (APVP) ese año (5). Si bien en la Primera Encuesta Argentina de Factores de Riesgo (6) se obtuvieron datos poblacionales de la prevalencia y la distribución de los factores de riesgo cardiovascular conocidos en la población adulta, no se conoce aún el efecto real, tanto sanitario como económico, que esos factores de riesgo tienen sobre la salud de la población argentina.

Este efecto de las enfermedades sobre la población, conocido como carga de la enfermedad, se mide en años de vida perdidos por muerte prematura (AVPMP) y años de vida saludable (AVISA) perdidos como indicador de los años vividos con discapacidad (7). Así, la carga de la enfermedad refleja no solamente las tasas de incidencia, prevalencia y mortalidad de una enfermedad, sino también el grado y la duración de la incapacidad y la reducción de la calidad de vida que provoca. De esta forma, los estudios que miden este indicador en una población dada constituyen una herramienta indispensable para que los encargados de tomar decisiones de salud pública asignen óptimamente los recursos y se logre el mayor beneficio.

El objetivo del presente estudio fue estimar la carga de la enfermedad, su proporción atribuible a los principales factores de riesgo cardiovascular modificables y los costos médicos directos por hospitalización, asociados con las enfermedades coronarias (infarto agudo del miocardio y angina inestable) y los accidentes cerebrovasculares en Argentina.

\section{MATERIALES Y MÉTODOS}

Se elaboró un modelo analítico a partir de los datos nacionales de prevalencia de los factores de riesgo cardiovascular obtenidos de la Primera Encuesta Argentina de Factores de Riesgo (6). En el presente estudio se analizaron todos los factores de riesgo cardiovascular medidos en esa encuesta a partir del autoinforme de los participantes: hipertensión arterial, hipercolesterolemia, sobrepeso, obesidad, hiperglucemia, tabaquismo actual y pasado, sedentarismo y consumo inadecuado de frutas y verduras.

Para este estudio sólo se consideraron las muertes registradas en Argentina en 2005, según la Clasificación estadística internacional de enfermedades y problemas relacionados con la salud, versión 10 (CIE-10) (8), como infarto agudo del miocardio (códigos I21, I22), angina ines- table (códigos I20, I24, I25) y accidente cerebrovascular (códigos I60-I61, I63-I64). Se excluyeron del análisis las muertes por insuficiencia cardíaca, ya que no todas podrían imputarse a eventos isquémicos coronarios.

Los riesgos relativos asociados con los factores de riesgo que se utilizaron en el modelo se tomaron de diversas fuentes bibliográficas (9-19) (cuadro 1).

El riesgo anual de muerte por las enfermedades cardiovasculares analizadas se calculó como la razón entre el número de muertes por cada causa — tomada del Registro de Defunciones de Argentina del año 2005- y el número de habitantes a mediados de ese año (20).

El riesgo anual de sufrir un evento cardiovascular (fatal o no fatal) por infarto agudo del miocardio, angina inestable o accidente cerebrovascular se calculó como la razón entre el riesgo de tener un evento fatal por una de esas enfermedades y su letalidad. El número de eventos no fatales ocurridos en Argentina en 2005 se calculó como la diferencia entre los eventos fatales registrados y el total estimado de eventos (fatales y no fatales). La letalidad se calculó como la razón entre el número específico de egresos por defunción debido a cada una de las causas estudiadas y el número total de egresos, según los datos de egresos hospitalarios del sector público en Argentina en el año 2000 (21).

Para calcular los AVISA se sumaron los años vividos con discapacidad (AVD) y los AVPMP.

Los AVD se calcularon mediante la siguiente ecuación (22):

$$
\begin{aligned}
\mathrm{AVD}= & D\left(( \frac { K C e ^ { r a } } { ( r + \beta ) ^ { 2 } } ) \left(e^{-(r+\beta)(L+a)}(-(r+\beta)\right.\right. \\
& (L+a)-1)-e^{-(r+\beta) a}(-(r+\beta) \\
& \left.a-1))+\left(\frac{(1-K)}{r}\right)\left(1-e^{-r L}\right)\right)
\end{aligned}
$$

dónde, $D$ es la ponderación de la discapacidad asociada con las enfermedades, según un estudio de carga de la enfermedad realizado en Australia (23); $K$ es el factor de modulación de la ponderación social por edad (valor entre 0 y 1 ); $\mathrm{C}$ es una constante con un valor calculado de 0,$1658 ; r$ es la tasa de descuento, tomada como 3\%; $a$ es la edad de inicio de la enfermedad; $\beta$, es el parámetro de la función de ponderación según la edad $(0,05)$; y $L$ es la duración de la enfermedad, expresada en años, estimada con el 


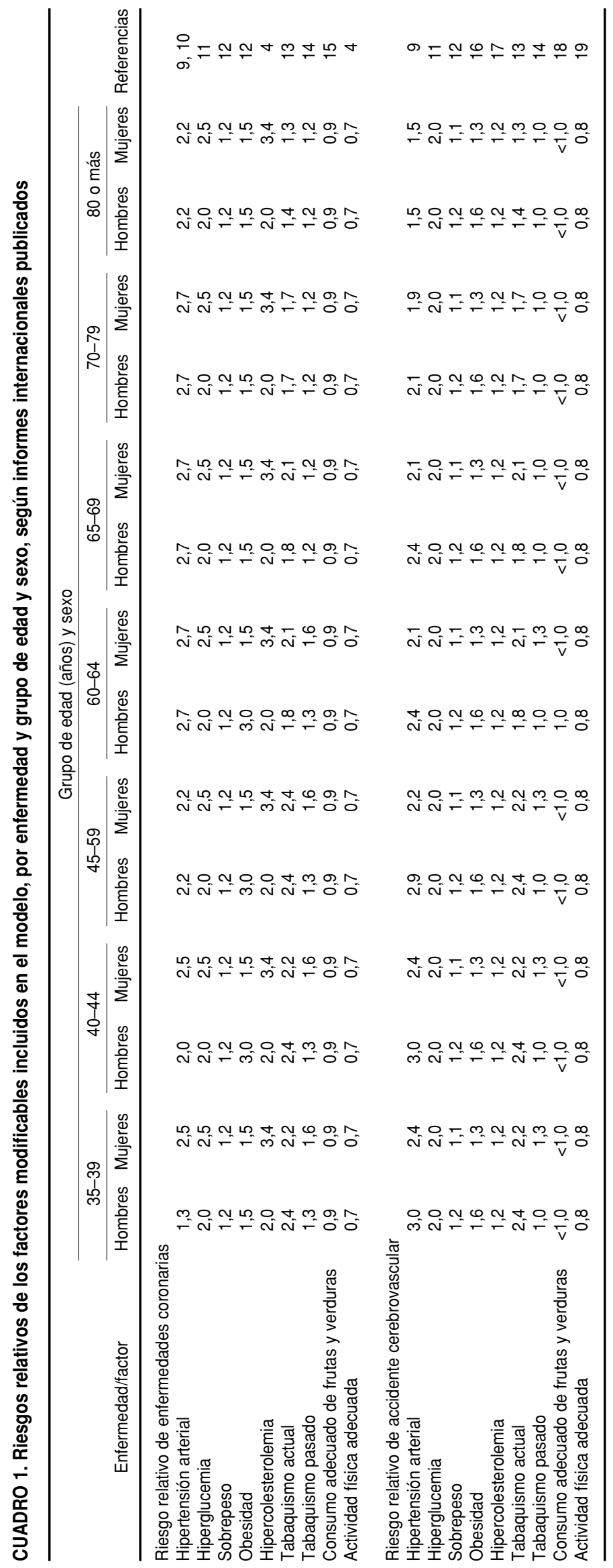


programa DISMOD II (24) y cotejada en un modelo ad hoc de Markov programado en Microsoft Excel ${ }^{\circledR}$.

Para calcular los AVD acumulados se multiplicó el valor obtenido en esta ecuación por la incidencia de eventos no fatales en cada grupo de edad y sexo.

Para el cálculo de los AVPMP se empleó la ecuación siguiente:

$$
\begin{aligned}
\mathrm{AVPMP}= & \left(( \frac { K C e ^ { r a } } { ( r + \beta ) ^ { 2 } } ) \left(e^{-(r+\beta)(E V E+a)}(-(r+\beta)\right.\right. \\
& (E V E+a)-1)-e^{-(r+\beta) a}(-(r+\beta) \\
& \left.a-1))+\left(\frac{(1-K)}{r}\right)\left(1-e^{-r E V E}\right)\right)
\end{aligned}
$$

dónde, $K$ es el factor de modulación de la ponderación social por edad (con valor entre 0 y 1); $C$ es una constante con un valor calculado de 0,1658 ; $r$ es la tasa de descuento, tomada como 3\%; $a$ es la edad de muerte; $\beta$ es el parámetro de la función de ponderación según la edad $(0,05)$; y EVE es la expectativa de vida estándar a la edad $a$, según las tablas internacionales de vida: 80 años para los hombres y 82,5 para las mujeres (22).

Para calcular los AVPMP acumulados se multiplicó el valor obtenido en esta ecuación por el número de muertes causadas por estas enfermedades en cada grupo de edad y sexo.

En cuanto a los APVP, se calcularon a partir de la expectativa de vida en Argentina según las tablas de vida locales (25).

Los costos de hospitalización por las enfermedades cardiovasculares analizadas se calcularon mediante la técnica de microcosteo - a partir de los costos promedio en los subsectores público, privado y de seguridad social- de cuatro tipos de recursos: días-cama, estudios y servicios auxiliares, medicamentos, y pruebas de laboratorio.

Los costos unitarios de los estudios y servicios auxiliares, los análisis de laboratorio y la hospitalización se tomaron de dos fuentes: la base de datos de costos sanitarios, elaborada por el Instituto de Efectividad Clínica y Sanitaria (26), y el nomenclador asistencial nacional y de aranceles para los hospitales públicos de gestión descentralizada, aprobado por el Ministerio de Salud (27). Los costos unitarios de los medicamentos se tomaron del Manual Farmacéutico (28) y de fuentes internas no publicadas del Programa Remediar, perteneciente al Ministerio de Salud de Argentina; se verificó el precio de las distintas presentaciones de las marcas comerciales disponibles para cada principio activo utilizado durante 2005. Para cada medicamento se estableció una unidad de dosis - a partir de la dosis diaria definida- y su correspondiente precio unitario; con esos datos se calculó la media aritmética de los precios unitarios. Los costos correspondieron a enero-febrero de 2007, según el rubro de atención médica y gastos para la salud del índice de precios al consumidor (29). Se estimó la tasa de uso de cada recurso médico o medicamento a partir de la proporción de personas con una enfermedad dada que utilizan ese recurso o medicamento, según los registros de pacientes (30-32) y la experiencia obtenida en el programa de protección integral general de los accidentes cerebrovasculares, PROTEGE-ACV, del Hospital Italiano de la Ciudad Autónoma de Buenos Aires (María Cristina Zurrú, comunicación personal, 2007). Como costo total de cada recurso identificado se tomó el producto de la multiplicación del precio unitario, el número de unidades consumidas y la tasa de uso correspondiente. Finalmente, para calcular el costo de cada evento agudo se sumaron los costos totales de cada recurso empleado.

Todos los costos se calcularon en pesos argentinos (\$) ajustados al primer bimestre de 2007 y se convirtieron a dólares estadounidenses (US\$) según la tasa de cambio de 2007 (US\$ 1,00 = \$3,0945) (33).

\section{Modelo de simulación epidemiológica}

Se elaboró un modelo de simulación de tipo matricial, con un núcleo programado en Microsoft Excel ${ }^{\circledR}$ en el que se almacenaron los datos o insumos requeridos por el modelo y se realizaron los cálculos de estimación central en forma determinística. En la matriz principal del modelo se introdujeron los datos de prevalencia por edad de las 288 posibles combinaciones de factores de riesgo; esos datos se obtuvieron de las 41392 observaciones contenidas en la base de datos de la Primera Encuesta Argentina de Factores de Riesgo. En el modelo sólo se utilizaron los datos de las personas mayores de 35 años por constituir este el grupo de mayor incidencia de enfermedades coronarias y accidentes cerebrovasculares y ser el grupo diana de las intervenciones clínicas para la reducción del riesgo cardiovascular.

Como riesgo relativo total para cada combinación de factores de riesgo en la población estudiada se consideró el producto de la multiplicación de los riesgos relativos individuales de cada uno de los factores presentes (34). La variabilidad en las distribuciones matriciales del modelo de simulación se programó en lenguaje Python (35). Para el análisis basal se utilizó un descuento de 3\% anual para los APVP y AVISA. Como análisis complementarios se calcularon los APVP y los AVISA sin descuento, así como los AVISA con ponderación social (con descuento y sin él).

Después de tener los estimados poblacionales (EP) del número de eventos fatales y no fatales, los APVP, los AVISA perdidos y los costos totales, se procedió a calcular la fracción atribuible poblacional (FAP) relacionada con los factores de riesgo modificables analizados, tanto en forma individual como combinada. Para ello, por cada conjunto de estimados poblacionales se calcularon los valores de la estimación alternativa (EA) que se obtendrían si el factor de riesgo índice empleado no contribuyera en realidad a aumentar el riesgo cardiovascular. La FAP se calculó mediante la siguiente fórmula:

$$
F A P=\frac{E P-E A}{E P}
$$

El intervalo de confianza de la FAP se calculó mediante el método de Monte Carlo de segundo orden. Para ello se realizaron 1000 simulaciones o iteraciones del análisis de carga de la enfermedad, en las que se modificó la contribución de cada factor de riesgo al total de eventos esperados, sin variar el número de eventos fatales y no fatales y los riesgos relativos de cada factor, sus costos y AVISA asociados.

Con el fin de evaluar el efecto que podría tener sobre la estimación poblacional final la falta de datos de presión arterial, glucemia y colesterol - ya que algunos participantes en la Primera Encuesta Argentina de Factores de Riesgo informaron que nunca se habían realizado esos estudios-, se hizo un análisis de imputación múltiple. Para ello se estimó el riesgo que tenía una persona de esta población de tener diabetes, hipertensión arterial o hipercolesterolemia, a partir de otras variables conocidas como la edad, el peso corporal y la presencia de otros factores de riesgo. Los nuevos valores de prevalencia obtenidos para las 288 combinaciones de factores de riesgo des- 
pués de la imputación se utilizaron como insumos del modelo epidemiológico. Para este análisis se utilizó el programa informático STATA versión 8.0.

\section{RESULTADOS}

Según datos oficiales, en 2005 murieron 89180 personas por problemas cardiovasculares en Argentina (30,6\% del total de defunciones); la causa de muerte más frecuente fue la insuficiencia cardíaca, con 28074 fallecidos. De las enfermedades evaluadas en este estudio, en 2005 se registraron en Argentina 39648 muertes $(14,8 \%$ del total) por infarto agudo del miocardio, angina inestable y accidentes cerebrovasculares en personas mayores de 35 años (54,0\% de ellas en hombres y $46,0 \%$ en mujeres); más de la mitad de esas muertes ( $7,6 \%$ del total) estaban asociadas con accidentes cerebrovasculares. La letalidad promedio de las enfermedades coronarias en personas mayores de 35 años fue de $11,9 \%$ en hombres y $18,1 \%$ en mujeres, mientras que para los accidentes cerebrovasculares fue de $17,4 \%$ y $18,9 \%$, respectivamente.

CUADRO 2. Costos de las enfermedades coronarias y los accidentes cerebrovasculares y carga de las enfermedades cardiovasculares según el sexo, Argentina, 2005

\begin{tabular}{|c|c|c|c|c|c|c|}
\hline \multirow[b]{2}{*}{ Indicador } & \multicolumn{2}{|c|}{ Hombres } & \multicolumn{2}{|c|}{ Mujeres } & \multicolumn{2}{|l|}{ Total } \\
\hline & No. & $\%$ & No. & $\%$ & No. & $\%$ \\
\hline \multicolumn{7}{|l|}{ Eventos agudos fatales y no fatales } \\
\hline Infarto agudo del miocardio & 62132 & 72,1 & 24031 & 27,9 & 86163 & 100,0 \\
\hline Angina inestable & 51660 & 68,5 & 23751 & 31,5 & 75411 & 100,0 \\
\hline Accidente cerebrovascular & 53432 & 52,7 & 48018 & 47,3 & 101450 & 100,0 \\
\hline Enfermedades cardiovasculares & 167225 & 63,6 & 95800 & 36,4 & 263025 & 100,0 \\
\hline Costo total ${ }^{\mathrm{a}}$ & 334457306 & 64,4 & 184717041 & 35,6 & 519174347 & 100,0 \\
\hline Años de vida saludable perdidos ${ }^{b}$ & 293419 & 48,0 & 318217 & 52,0 & 611636 & 100,0 \\
\hline Años potenciales de vida perdidos ${ }^{c}$ & 218547 & 55,4 & 175617 & 44,6 & 394164 & 100,0 \\
\hline
\end{tabular}

a Se consideraron solo los costos médicos directos por hospitalización. Los costos se calcularon en pesos argentinos (\$) ajustados al primer bimestre de 2007 y se convirtieron a dólares estadounidenses (US\$) según la tasa de cambio de 2007 (US $\$ 1,00=\$ 3,0945)(33)$.

bPor infarto agudo del miocardio, angina inestable y accidente cerebrovascular. Se calculó con un descuento de 3,0\% anual, sin ponderación social.

c Por infarto agudo del miocardio, angina inestable y accidente cerebrovascular. Se calculó con un descuento de 3,0\% anual.

CUADRO 3. Costos de hospitalización por cada evento cardiovascular agudo, Argentina, 2005

\begin{tabular}{|c|c|c|c|c|}
\hline \multirow[b]{2}{*}{ Concepto } & \multicolumn{2}{|c|}{ Enfermedades coronarias agudas ${ }^{a}$} & \multicolumn{2}{|c|}{ Accidentes cerebrovasculares } \\
\hline & Costo $(U S \$)^{b}$ & $\%$ & Costo (US\$) & $\%$ \\
\hline Camas-día & 1267,25 & 59,6 & 1267,25 & 73,2 \\
\hline Estudios y servicios auxiliares ${ }^{c}$ & 415,54 & 19,5 & 195,95 & 11,3 \\
\hline Medicamentos & 388,50 & 18,3 & 233,14 & 13,5 \\
\hline Laboratorio & 55,18 & 2,6 & 34,47 & 2,0 \\
\hline Total & 2126,47 & 100,0 & 1730,81 & 100,0 \\
\hline
\end{tabular}

a Por infarto agudo del miocardio y angina inestable.

'Los costos se calcularon en pesos argentinos (\$) ajustados al primer bimestre de 2007 y se convirtieron a dólares estadounidenses (US\$) según la tasa de cambio de 2007 (US\$1,00 = \$3,0945) (33).

'Por consultas médicas y procedimientos diagnósticos y terapéuticos especializados. eventos agudos analizados y más de un tercio de los AVISA perdidos por enfermedades coronarias y accidentes cerebrovasculares en Argentina en 2005 (cuadro 5). La hipercolesterolemia fue el segundo factor de riesgo cardiovascular más importante entre las mujeres $(23,8 \%)$, pero el segundo menos importante entre los hombres $(14,7 \%)$. El riesgo cardiovascular derivado del consumo inadecuado de frutas y verduras fue pequeño (cuadro 5).

Los resultados del análisis de sensibilidad probabilístico no mostraron diferencias importantes en la FAP, ya fuera con el descuento de $3 \%$ anual o sin él, e independientemente de la ponderación social (cuadro 6).

Las diferencias entre los valores de la FAP obtenidos antes y después del proceso de imputación de la información faltante en la base de datos de la Primera Encuesta Argentina de Factores de Riesgo resultaron muy pequeñas (menos de 2,2\% en todos los casos), por lo que se tomaron los resultados sin imputaciones. Con la aplicación del descuento anual de $3 \%$ en los AVISA perdidos y los APVP se redujo ligeramente el valor de la FAP con respecto al calculado sin el descuento; la ponderación social mostró un efecto heterogéneo sobre la FAP estimada, ya que disminuyó en los hombres y aumentó en las mujeres (cuadro 6).

\section{DISCUSIÓN}

Más de $70 \%$ de los más de 600000 AVISA perdidos y de los casi 400000 APVP contabilizados en Argentina en 2005 debido a las enfermedades cardiovasculares estudiadas se pueden atribuir a factores de riesgo cardiovascular modificables, por lo que deben considerarse eventos evitables.

La hipertensión arterial fue el factor de riesgo individual más importante, ya que estuvo asociada con más de la tercera parte de los eventos cardiovasculares y de los AVISA perdidos.

Al bajo consumo de frutas y verduras, por su parte, correspondió una baja FAP. Esto coincide con afirmaciones de que el consumo de frutas y verduras es solo un factor dentro de la alimentación saludable y quizá no el más importante (36). Sin embargo, esto no significa que las intervenciones destinadas a elevar el consumo de frutas y verduras no sean necesarias o que no sean efectivas en función 
CUADRO 4. Proporción de la carga de las enfermedades cardiovasculares atribuible a factores de riesgo modificables, Argentina, 2005

\begin{tabular}{|c|c|c|c|c|c|c|}
\hline \multirow[b]{2}{*}{ Indicador } & \multicolumn{2}{|c|}{ Hombres } & \multicolumn{2}{|c|}{ Mujeres } & \multicolumn{2}{|c|}{ Total } \\
\hline & (\%) & IC95\% ${ }^{a}$ & $(\%)$ & IC95\% & (\%) & IC95\% \\
\hline \multicolumn{7}{|c|}{ Total de eventos agudos fatales y no fatales } \\
\hline Infarto agudo del miocardio & 82,4 & $82,0-82,8$ & 84,4 & $83,2-85,3$ & 83,0 & $82,5-83,4$ \\
\hline Angina inestable & 82,1 & $81,6-82,5$ & 83,8 & $82,2-85,0$ & 82,6 & $82,0-83,1$ \\
\hline Accidente cerebrovascular & 67,0 & $66,1-67,8$ & 58,0 & $56,8-59,1$ & 62,8 & $62,0-63,4$ \\
\hline Enfermedades cardiovasculares & 77,4 & $76,8-77,9$ & 71,0 & $69,8-72,1$ & 75,1 & $74,5-75,6$ \\
\hline Costo total ${ }^{b}$ & 78,0 & $77,5-78,5$ & 72,4 & $71,1-73,5$ & 76,0 & $75,4-76,5$ \\
\hline Años de vida saludable perdidos ${ }^{C}$ & 76,5 & $76,1-76,9$ & 66,2 & $65,5-66,9$ & 71,1 & $70,7-71,6$ \\
\hline Años potenciales de vida perdidos ${ }^{d}$ & 77,0 & $76,5-77,4$ & 70,0 & $69,0-70,9$ & 73,9 & $73,3-74,3$ \\
\hline
\end{tabular}

alC95\%: intervalo de confianza de 95\%.

b Se consideraron solo los costos médicos directos por hospitalización. Los costos se calcularon en pesos argentinos (\$) ajustados al primer bimestre de 2007 y se convirtieron a dólares estadounidenses (US\$) según la tasa de cambio de 2007 (US\$1,00 = \$3,0945) (33).

cPor infarto agudo del miocardio, angina inestable y accidentes cerebrovasculares Se calculó con un descuento de 3,0\% anual, sin ponderación social.

dPor infarto agudo del miocardio, angina inestable y accidentes cerebrovasculares, con un descuento de 3,0\% anual.

del costo. La relación costo-efectividad de una intervención depende no solo de la eficacia de la medida en la reducción del riesgo de los eventos adversos, sino también del costo de la intervención.
Entre 1994 y 2004 se han publicado estudios nacionales sobre carga de enfermedad en Chile (37), Ecuador (38), México (39) y Perú (40); los resultados de un estudio similar realizado recientemente en Argentina aún no están disponibles. A pesar de las diferencias metodológicas, la variabilidad en los AVISA perdidos por enfermedades cardiovasculares informados en esos países (desde aproximadamente $6 \%$ en Perú hasta $11 \%$ en Chile) es probablemente un reflejo de los distintos momentos de la transición epidemiológica en que se encontraban esos países en el momento del estudio.

Según Ezzati y colaboradores (41), la carga anual de las enfermedades cardiovasculares atribuible a la hipertensión arterial, la hipercolesterolemia, el sobrepeso y la obesidad en países latinoamericanos con baja mortalidad en niños y adultos - como Argentina- asciende a 5,5 millones de AVISA perdidos en hombres y 4,6 millones en mujeres, equivalentes a 23304 AVISA perdidos por 100000 habitantes en 10 años. Con una población estimada de 37282970 en 2005 en Argentina, según las proyecciones del último censo nacional de 2001 (42), a estos tres factores de riesgo corres-

CUADRO 5. Proporción del costo y la carga de las enfermedades cardiovasculares atribuible a los factores de riesgo analizados, según el sexo, Argentina, 2005

\begin{tabular}{|c|c|c|c|c|c|c|c|}
\hline Grupo/indicador & $\begin{array}{l}\text { Tabaquismo } \\
\text { actual y } \\
\text { pasado, } \\
\%(\text { IC95\%) }\end{array}$ & $\begin{array}{l}\text { Sobrepeso } \\
\text { y obesidad, } \\
\%(I C 95 \%)\end{array}$ & $\begin{array}{l}\text { Hipertensión } \\
\text { arterial, } \\
\% \text { (IC95\%) }\end{array}$ & $\begin{array}{c}\text { Hiper- } \\
\text { colesterolemia, } \\
\%(\mathrm{IC} 95 \%)\end{array}$ & $\begin{array}{l}\text { Hiperglucemia, } \\
\% \text { (IC95\%) }\end{array}$ & $\begin{array}{l}\text { Sedentarismo, } \\
\%(\mathrm{IC} 95 \%)\end{array}$ & $\begin{array}{c}\text { Consumo } \\
\text { inadecuado de } \\
\text { frutas y verduras, } \\
\%(\text { IC } 95 \%)\end{array}$ \\
\hline \multicolumn{8}{|l|}{ Hombres } \\
\hline \multicolumn{8}{|c|}{ Total de eventos agudos fatales y no fatales } \\
\hline Infarto agudo del miocardio & $26,7(26,2-27,1)$ & $24,7(24,1-25,2)$ & $37,0(36,4-37,6)$ & $19,0(18,3-19,6)$ & $12,4(11,8-13,0)$ & $20,2(19,8-20,6)$ & $3,8(3,7-3,9)$ \\
\hline Angina inestable & $23,1(22,6-23,5)$ & $22,3(21,8-22,8)$ & $40,1(39,3-40,8)$ & $19,3(18,6-20,0)$ & $13,5(12,8-14,2)$ & $20,6(20,2-21,2)$ & $3,5(3,3-3,7)$ \\
\hline Accidente cerebrovascular & $14,2(13,7-14,7)$ & $16,7(16,0-17,3)$ & $35,2(34,3-36,0)$ & $5,2(4,9-5,5)$ & $13,5(12,6-14,4)$ & $12,2(11,7-12,6)$ & $1,4(1,3-1,5)$ \\
\hline Enfermedades cardiovasculares & $21,6(21,1-22,0)$ & $21,4(20,9-21,9)$ & $37,4(36,7-38,1)$ & $14,7(14,1-15,2)$ & $13,1(12,4-13,8)$ & $17,8(17,4-18,2)$ & $2,9(2,8-3,1)$ \\
\hline Costo total ${ }^{b}$ & $22,0(21,6-22,5)$ & $21,7(21,2-22,2)$ & $37,6(36,8-38,2)$ & $15,2(14,7-15,8)$ & $13,1(12,4-13,8)$ & $18,1(17,7-18,6)$ & $3,0(2,9-3,2)$ \\
\hline Años de vida perdidos ${ }^{c}$ & $22,1(21,6-22,6)$ & $20,5(20,1-20,9)$ & $38,7(38,1-39,3)$ & $12,1(11,7-12,6)$ & $13,0(12,3-13,7)$ & $15,6(15,3-16,0)$ & $2,5(2,4-2,6)$ \\
\hline Años potenciales de vida perdidos ${ }^{d}$ & $21,6(21,1-22,1)$ & $20,7(20,2-21,1)$ & $38,7(38,1-39,3)$ & $13,4(12,9-13,8)$ & $13,1(12,4-13,8)$ & $16,5(16,2-16,9)$ & $2,7(2,6-2,8)$ \\
\hline \multicolumn{8}{|l|}{ Mujeres } \\
\hline \multicolumn{8}{|c|}{ Total de eventos agudos fatales y no fatales } \\
\hline Infarto agudo del miocardio & $11,9(11,5-12,3)$ & $11,0(10,5-11,6)$ & $42,4(41,1-43,5)$ & $41,0(39,2-42,7)$ & $17,7(16,2-19,1)$ & $22,6(22,2-23,1)$ & $2,3(2,2-2,5)$ \\
\hline Angina inestable & $8,5(8,0-9,0)$ & $10,5(9,9-11,2)$ & $42,5(40,7-44,0)$ & $41,1(38,4-43,1)$ & $17,7(15,8-19,6)$ & $23,7(23,1-24,2)$ & $2,2(2,0-2,4)$ \\
\hline Accidente cerebrovascular & $7,1(6,8-7,6)$ & $6,1(5,8-6,5)$ & $29,9(28,9-30,8)$ & $6,6(6,1-7,1)$ & $12,8(11,5-14,2)$ & $14,0(13,6-14,3)$ & $1,0(0,9-1,1)$ \\
\hline Enfermedades cardiovasculares & $8,6(8,3-9,1)$ & $8,4(8,0-8,9)$ & $36,2(34,9-37,3)$ & $23,8(22,4-24,9)$ & $15,2(13,8-16,8)$ & $18,6(18,1-19,0)$ & $1,6(1,5-1,8)$ \\
\hline Costo total ${ }^{\mathrm{b}}$ & $8,8(8,4-9,3)$ & $8,7(8,2-9,7)$ & $36,8(35,5-37,9)$ & $25,5(24,1-26,7)$ & $15,5(14,0-17,0)$ & $19,0(18,6-19,5)$ & $1,7(1,5-1,9)$ \\
\hline Años de vida perdidos ${ }^{c}$ & $10,5(10,1-10,9)$ & $7,6(7,3-7,9)$ & $34,5(33,8-35,2)$ & $14,5(13,9-15,1)$ & $14,1(13,2-15,1)$ & $15,3(15,0-15,6)$ & $1,3(1,3-1,4)$ \\
\hline Años potenciales de vida perdidos ${ }^{d}$ & $10,4(10,0-10,8)$ & $8,3(7,9-8,7)$ & $36,0(35,0-36,9)$ & $20,7(19,7-21,6)$ & $14,8(13,6-16,0)$ & $17,3(16,9-17,6)$ & $1,6(1,5-1,7)$ \\
\hline \multicolumn{8}{|l|}{ Total } \\
\hline \multicolumn{8}{|c|}{ Total de eventos agudos fatales y no fatales } \\
\hline Infarto agudo del miocardio & $22,5(22,2-22,8)$ & $20,9(20,4-21,3)$ & $38,5(37,9-39,1)$ & $25,1(24,4-25,8)$ & $13,9(13,2-14,5)$ & $20,9(20,6-21,2)$ & $3,4(3,3-3,5)$ \\
\hline Angina inestable & $18,5(18,1-18,8)$ & $18,6(18,2-19,0)$ & $40,9(40,1-41,5)$ & $26,2(25,2-27,0)$ & $14,8(14,0-15,6)$ & $21,6(21,2-22,0)$ & $3,1(3,0-3,2)$ \\
\hline Accidente cerebrovascular & $10,8(10,5-11,2)$ & $11,7(11,3-12,0)$ & $32,7(32,0-33,3)$ & $5,8(5,6-6,1)$ & $13,2(12,3-14,0)$ & $13,0(12,7-13,3)$ & $1,2(1,1-1,3)$ \\
\hline Enfermedades cardiovasculares & $16,9(16,5-17,2)$ & $16,7(16,3-17,0)$ & $37,0(36,3-37,5)$ & $18,0(17,4-18,5)$ & $13,9(13,1-14,6)$ & $18,1(17,7-18,3)$ & $2,5(2,4-2,6)$ \\
\hline Costo total ${ }^{b}$ & $17,3(17,0-17,6)$ & $17,1(16,7-17,4)$ & $37,3(36,6-37,9)$ & $18,9(18,3-19,5)$ & $13,9(13,2-14,7)$ & $18,4(18,1-18,7)$ & $2,6(2,5-2,7)$ \\
\hline AVISA perdidos totales ${ }^{c}$ & $16,1(15,7-16,4)$ & $13,8(13,5-14,0)$ & $36,6(36,1-37,0)$ & $13,4(13,0-13,7)$ & $13,6(13,0-14,2)$ & $15,5(15,2-15,7)$ & $1,9(1,8-2,0)$ \\
\hline Años potenciales de vida perdidos ${ }^{d}$ & $16,6(16,3-16,9)$ & $15,1(14,8-15,4)$ & $37,5(36,9-38,0)$ & $16,6(16,1-17,1)$ & $13,9(13,2-14,6)$ & $16,9(16,6-17,1)$ & $2,2(2,1-2,3)$ \\
\hline
\end{tabular}

a IC95\%: intervalo de confianza de 95\%.

${ }^{\text {b }}$ Se consideraron solo los costos médicos directos por hospitalización. Los costos se calcularon en pesos argentinos (\$) ajustados al primer bimestre de 2007 y se convirtieron a dólares estadounidenses (US\$) según la tasa de cambio de 2007 (US\$ 1,00 = \$3,0945) (33).

cPor infarto agudo del miocardio, angina inestable y accidentes cerebrovasculares. Se calculó con un descuento de 3,0\% anual, sin ponderación social.

dPor infarto agudo del miocardio, angina inestable y accidentes cerebrovasculares, con un descuento de $3,0 \%$ anual. 
CUADRO 6. Resultados del análisis de sensibilidad de los cálculos de la carga de las enfermedades cardiovasculares y la fracción atribuible poblacional (FAP) relacionada con el conjunto de factores de riesgo modificables analizados, Argentina, 2005

\begin{tabular}{|c|c|c|c|c|c|c|c|c|c|}
\hline \multirow[b]{2}{*}{ Indicador/forma de cálculo } & \multicolumn{3}{|c|}{ Hombres } & \multicolumn{3}{|c|}{ Mujeres } & \multicolumn{3}{|c|}{ Total } \\
\hline & Años & FAP $(\%)$ & $\operatorname{IC} 95 \%^{a}$ & Años & FAP (\%) & IC95\% & Años & FAP (\%) & IC95\% \\
\hline \multicolumn{10}{|l|}{ Años potenciales de vida perdidos } \\
\hline Con descuento ${ }^{b}$ & 218547 & 77,0 & $76,5-77,4$ & 175617 & 70,0 & $69,0-70,9$ & 394164 & 73,9 & $73,3-74,3$ \\
\hline \multicolumn{10}{|l|}{ Años de vida saludable } \\
\hline Sin ponderación social, sin descuento & 368141 & 77,0 & $76,6-77,4$ & 400885 & 66,7 & $66,1-67,4$ & 769026 & 71,6 & $71,3-72,0$ \\
\hline Sin ponderación social, con descuentob & 293419 & 76,5 & $76,1-76,9$ & 318217 & 66,2 & $65,5-66,9$ & 611636 & 71,1 & $70,7-71,6$ \\
\hline
\end{tabular}

aIC95\%: intervalo de confianza de 95\%.

${ }^{\text {b}}$ Descuento de 3,0\% anual.

pondería $63,7 \%$ de la carga de las enfermedades cardiovasculares estimada en el presente estudio. Esto equivale aproximadamente a 390000 AVISA perdidos en un año y 10460 AVISA perdidos por 100000 habitantes por año, es decir, menos de la mitad de lo publicado para países como Argentina en el estudio citado (42). Esta diferencia podría deberse a que en ese estudio se estimó la carga de la enfermedad en los países de América Latina a partir de inferencias, mientras que en el presente se precisaron los estimados para Argentina a partir de datos específicos nacionales obtenidos en una encuesta nacional de factores de riesgo, calibrados con las estadísticas de la mortalidad por enfermedades coronarias y accidentes cerebrovasculares en 2005. También es posible que Ezzati y colaboradores no hayan tomado en cuenta la reducción en la incidencia ajustada de las enfermedades cardiovasculares, evidenciada en Argentina entre 1970 y 2000 (43).

En el presente estudio no se tomaron en cuenta las muertes por insuficiencia cardíaca, que constituyen la primera causa de muerte por problemas cardiovasculares en Argentina; se estima que entre $36 \%$ y $44 \%$ de los pacientes argentinos con este trastorno presentan antecedentes de cardiopatía isquémica (44, 45). Aunque esto podría llevar a subestimar la carga de las enfermedades cardiovasculares atribuible a enfermedades coronarias, la imputación a esta enfermedad de las muertes por insuficiencia cardíaca es un proceso complejo y cuestionable, por lo que en la presente investigación se mantuvo un enfoque conservador en este sentido. A esto se puede añadir que en un estudio reciente se demostró que en Argentina se confunde en ocasiones la insuficiencia car- díaca como causa de muerte con otros diagnósticos imprecisos de muerte por problemas cardiovasculares (como la ateromatosis), lo que podría llevar a cierto subregistro de esta enfermedad en los certificados de defunción, aunque no se hayan observado muertes por isquemia coronaria aguda entre estos diagnósticos imprecisos (46).

Es importante reconocer algunas otras limitaciones del presente estudio: 1) algunos datos, como los riesgos relativos, se tomaron de fuentes internacionales, dada la falta de estudios prospectivos observacionales en Argentina y América Latina; 2) se consideró que los riesgos relativos de las enfermedades coronarias agudas y los accidentes cerebrovasculares eran independientes $-\mathrm{y}$, por lo tanto, multiplicativos-, lo que, si bien no es necesariamente un hecho, es un enfoque ampliamente utilizado en modelos predictivos de riesgo cardiovascular $(47) ; 3)$ no se consideró en el análisis de sensibilidad la incertidumbre sobre los costos y otros parámetros epidemiológicos, como los riesgos relativos, la letalidad y la tasa de mortalidad; 4) la estimación de la tasa de letalidad se basó solamente en los datos de egresos hospitalarios del sector público, que podría diferir de la del sector privado o de la seguridad social, si bien no hay evidencias empíricas que sustenten este hecho; 5) no se estimaron por separado los costos del infarto agudo del miocardio y la angina inestable debido a que el objetivo era estimar el costo total atribuido a enfermedades coronarias agudas; $y$ 6) como en todos los estudios que emplean modelos basados en datos secundarios, para el presente se compendiaron datos provenientes de diferentes fuentes y se partió de diversos supues- tos, tanto en el diseño como en el análisis de los resultados.

Se debe destacar que, si bien los datos de prevalencia empleados en el presente estudio se obtuvieron por medio del autoinforme, el proceso de imputación múltiple de valores faltantes de hipertensión arterial, hipercolesterolemia e hiperglucemia no modificó significativamente los valores de la FAP de esos factores de riesgo, por lo que los resultados obtenidos se pueden considerar robustos.

Aún cuando las enfermedades cardiovasculares son la primera causa de muerte y discapacidad en Argentina, los programas nacionales del Ministerio de Salud, así como de los ministerios y secretarías provinciales y municipales continúan centrando sus intervenciones casi exclusivamente en el control de las enfermedades transmisibles o perinatales mediante diversos programas verticales. Se deben emprender acciones y programas dirigidos a cambiar los hábitos y estilos de vida de la población e implementar intervenciones clínicas que lleven a reducir la incidencia de las enfermedades cardiovasculares en Argentina. En el marco del Proyecto de Priorización para el Control de Enfermedades, del Banco Mundial, se han identificado varias intervenciones efectivas en función del costo dirigidas a la prevención y el tratamiento de las enfermedades crónicas con costos estimados de US\$ 1000 por AVISA evitado (48). En esta línea de trabajo, un estudio publicado recientemente que evaluó intervenciones clínicas y poblacionales implementadas para prevenir las afecciones cardiovasculares en la ciudad de Buenos Aires concluyó que, aunque menos efectivas en función del costo, esas intervenciones eran abso- 
lutamente asequibles para un país de medianos ingresos como Argentina (49).

Si en Argentina aún no se han implementado muchas intervenciones eficaces, efectivas y rentables dirigidas a prevenir las enfermedades cardiovasculares no es necesariamente por falta de recursos, sino en buena medida porque aún no se reconoce el efecto sanitario, económico y social de la epidemia de enfermedades crónicas en general, y de las cardiovasculares en particular (50).

En conclusión, en Argentina se pierden anualmente más de 600000 AVISA y se contabilizan casi 400000 APVP por enfermedades coronarias (infarto agudo del miocardio y angina inestable) y accidentes cerebrovasculares. Esto representa un costo adicional para Argentina de casi 520 millones de dólares estadounidenses al año. En general, 71,1\% de los AVISA perdidos, 73,9\% de los APVP, así como $76,0 \%$ de los costos asociados, son atribuibles a factores de riesgo modificables.

La mayor parte de la carga de la enfermedad en Argentina por enfermedades cardiovasculares está relacionada con factores de riesgo modificables - por lo tanto evitables- y podría reducirse mediante intervenciones poblacionales $\mathrm{y}$ clínicas basadas en un enfoque de riesgo, que ya han demostrado ser efectivas en función del costo, asequibles y factibles en países como Argentina.

Los datos presentados en este trabajo sobre la carga de las enfermedades car- diovasculares en Argentina deben contribuir a tomar decisiones informadas en la priorización y la asignación de recursos para enfrentar esta nueva epidemia, mediante intervenciones, ya sean dirigidas a toda la población o a grupos específicos de la población con mayor riesgo.

Agradecimiento. El presente trabajo de investigación se realizó con el apoyo de una beca para estudios colaborativos multicéntricos otorgada por el Ministerio de Salud de Argentina a través de la Comisión Nacional Salud Investiga. Lisandro Colantonio recibió una beca de Fogarty International Clinical Research para el período 2009-2010.

\section{REFERENCIAS}

1. World Health Organization. The World Health Report 2002. Reducing risks, promoting healthy life. Geneva: WHO; 2002. Hallado en http://www.who.int/entity/whr/2002/ en/whr02_en.pdf. Acceso el 10 de marzo de 2010.

2. Mathers CD, Lopez A, Stein C, Fat DM, Rao $\mathrm{C}$, Inoue $\mathrm{M}$, et al. Deaths and disease burden by cause: global burden of disease estimates for 2001 by World Bank Country Groups. Bethesda, MD: World Bank; Disease Control Priorities Project; 2005 (Working Paper No. 18, revised January 2005.)

3. World Health Organization. Preventing chronic diseases: a vital investment. Geneva: WHO; 2005. Hallado en http://www.who. int/chp/chronic_disease_report/contents/fo reword.pdf. Acceso el 10 de marzo de 2010.

4. Lanas F, Avezum A, Bautista LE, Díaz R, Luna M, Islam $S$, et al. Risk factors for acute myocardial infarction in Latin America: the INTERHEART Latin American study. Circulation. 2007;115(9):1067-74.

5. República Argentina, Ministerio de Salud y Ambiente. Argentina: indicadores básicos 2005. Buenos Aires: Ministerio de Salud y Ambiente; 2005. Hallado en http://www. bvs.org.ar/pdf/indicadores2005.pdf. Acceso el 28 de diciembre de 2009.

6. República Argentina, Ministerio de Salud. Primera Encuesta Nacional de Factores de Riesgo. Buenos Aires: Ministerio de Salud; 2006. Hallado en http://www.msal.gov.ar/ $\mathrm{htm} /$ Site/enfr/index.asp. Acceso el $10 \mathrm{de}$ marzo de 2010.

7. World Bank. World development report: investing in health. New York: Oxford University Press; 1993.

8. World Health Organization. 1CD-10: International statistical classification of diseases and related health problems, 10th revision. 2nd ed. Vol. 2. Geneva: WHO; 2004. Hallado en http://www.who.int/classifications/icd/IC D-10_2nd_ed_volume2.pdf. Acceso el 20 de marzo de 2010 .
9. Lewington S, Clarke R, Qizilbash N, Peto R, Collins R. Age-specific relevance of usual blood pressure to vascular mortality: a metaanalysis of individual data for one million adults in 61 prospective studies. Lancet. 2002;360(9349):1903-13.

10. Lloyd-Jones DM, Evans JC, Levy D. Hypertension in adults across the age spectrum: current outcomes and control in the community. J Am Med Assoc. 2005;294(4): 466-72.

11. Huxley R, Barzi F, Woodward M. Excess risk of fatal coronary heart disease associated with diabetes in men and women: metaanalysis of 37 prospective cohort studies. $\mathrm{Br}$ Med J. 2006;332(7533):73-8.

12. Bogers RP, Bemelmans WJ, Hoogenveen RT, Boshuizen HC, Woodward M, Knekt P, et al. Association of overweight with increased risk of coronary heart disease partly independent of blood pressure and cholesterol levels: a meta-analysis of 21 cohort studies including more than 300000 persons. Arch Intern Med. 2007;167(16):1720-8.

13. Ezzati M, López AD. Smoking and oral tobacco use. En: Ezzati M, López AD, Rodgers A, Murray CJL, ed. Comparative quantification of health risks: global and regional burden of disease attributable to selected major risk factors. Geneva: World Health Organization; 2004:883-957.

14. Smoking-attributable mortality, morbidity, and economic costs (SAMMEC). Adult SAMMEC - relative risk. Atlanta, GA: Centers for Disease Control and Prevention; sin año. Hallado en https://apps.nccd.cdc.gov/sammec/ edit_risk_data.asp. Acceso el 20 de marzo de 2010.

15. He FJ, Nowson CA, MacGregor GA. Fruit and vegetable consumption and stroke: metaanalysis of cohort studies. Lancet. 2006;367 (9507):320-6.

16. Hu G, Tuomilehto J, Silventoinen K, Sarti C, Mannisto S, Jousilahti P. Body mass index, waist circumference, and waist-hip ratio on the risk of total and type-specific stroke. Arch Intern Med. 2007;167(13):1420-7.

17. Amarenco $P$, Labreuche $J$, Lavallee $P$, Touboul PJ. Statins in stroke prevention and carotid atherosclerosis: systematic review and up-to-date meta-analysis. Stroke. 2004;35 (12):2902-9.

18. Dauchet L, Amouyel P, Dallongeville J. Fruit and vegetable consumption and risk of stroke: a meta-analysis of cohort studies. Neurology. 2005;65(8):1193-7.

19. Lee CD, Folsom AR, Blair SN. Physical activity and stroke risk: a meta-analysis. Stroke. 2003;34(10):2475-81.

20. República Argentina, Ministerio de Salud y Ambiente, Secretaría de Políticas, Regulación y Relaciones Sanitarias, Dirección de Estadísticas e Información de Salud. Estadísticas vitales, información básica 2005. Buenos Aires: Ministerio de Salud y Ambiente; 2006.

21. República Argentina, Ministerio de Salud, Secretaría de Políticas, Regulación y Relaciones Sanitarias. Egresos de establecimientos oficiales según variables seleccionadas. Buenos Aires: Ministerio de Salud; 2000.

22. Murray CJL, López AD. The global burden of disease: a comprehensive assessment of mortality and disability from diseases, injuries, and risk factors in 1990 and projected to 2020. Cambridge, MA: Harvard University Press; 1996.

23. Australia, Victorian Government Department of Human Services. Victorian Burden of Disease Study. Mortality and morbidity in 2001. Melbourne: Victorian Government Department of Human Services; 2005. Hallado en http://www.health.vic.gov.au/healthsta tus/downloads/bod_2001.pdf. Acceso el 10 de marzo de 2010.

24. Barendregt JJ, Van Oortmarssen GJ, Vos T, Murray CJ. A generic model for the assessment of disease epidemiology: the computational basis of DisMod II. Popul Health Metr. 2003;1(1):4

25. República Argentina, Instituto Nacional de Estadística y Censos. Tablas abreviadas de 
mortalidad 2000-2001. Total país y provincias. Buenos Aires: Instituto Nacional de Estadística y Censos; 2005.

26. Pichón-Rivière $\mathrm{A}$, Regueiro $\mathrm{A}$, Souto $\mathrm{A}$, Augustovski F. Base de datos de costos sanitarios argentinos. Buenos Aires: Instituto de Efectividad Clínica y Sanitaria; 2004. Hallado en http://www.iecs.org.ar/iecs-visor-publi cacion.php? cod_publicacion $=146 \&$ familia $=8 \&$ origen_publicacion $=$ buscador . Acceso el 20 de febrero de 2010.

27. República Argentina, Ministerio de Salud, Superintendencia de Servicios de Salud. Resolución 488/2002. Normas y módulos para los hospitales públicos de gestión descentralizada. Unidad Hospital Público (UHP). Buenos Aires: Ministerio de Salud; 2002.

28. Manual Farmacéutico en Línea. Buenos Aires: Editorial Alfa Beta; sin año. Hallado en http://www.alfabeta.net/mf-precios.xtp. Acceso el 13 de marzo de 2010.

29. República Argentina, Instituto Nacional de Estadísticas y Censos. Índice de precios al consumidor, Gran Buenos Aires. Hallado en http://www.indec.mecon.gov.ar/nueva web/cuadros/10/sh_ipc_2008.xls. Acceso el 10 de marzo de 2010.

30. Sposato LA, Esnaola MM, Zamora R, Zurrú MC, Fustinoni O, Saposnik G, et al. Quality of ischemic stroke care in emerging countries: the Argentinean National Stroke Registry (ReNACer). Stroke. 2008;(11):3036-41.

31. Sociedad Argentina de Cardiología. Encuesta de cardiopatía isquémica de la Sociedad Argentina de Cardiología. Buenos Aires: Sociedad Argentina de Cardiología; 2007.

32. República Argentina, Ministerio de Salud. Proyecto piloto de implementación de grupos relacionados por el diagnóstico (GRDs) del Ministerio de Salud. Buenos Aires: Ministerio de Salud; 2007.

33. Banco Central de la República Argentina. Estadísticas cambiarias. Buenos Aires: Banco Central; sin año. Hallado en http://www. bcra.gov.ar/estadis/es030101.asp. Acceso el 10 de marzo de 2010

34. Wilson PW, D'Agostino RB, Levy D, Belanger AM, Silbershatz H, Kannel WB. Prediction of coronary heart disease using risk factor categories. Circulation. 1998;97(18):1837-47.

35. Bassi S. A primer on Python for life science researchers. PLoS Comput Biol. 2007;3(11):e199.

36. MacDonald J, Brevard PB, Lee RE, Wagner T. Link between diet and cardiovascular disease in Latin America and the Caribbean using geographic information systems. Rev Panam Salud Publica. 2009;26(4):290-8.

37. Barrientos MC, Paz Aguilera Sanhueza $X$, Salas Vergara J. La carga de enfermedad en Chile. Informe final. Santiago: Ministerio de Salud Pública; 1996. Hallado en http:/ / epi.minsal. cl/ epi/html/sdesalud/carga/Inffin-carga-enf. pdf\#search $=\% 22$ carga $\% 20$ de $\% 20$ enferme dad\%20chile\%22. Acceso el 14 de marzo de 2010.

38. Lozada P, Aguinaga L, Páez R, Olmedo C, Pozo A. El peso de la enfermedad en el Ecuador. Hallado en http://www.opsecu. org/bevestre/revistas/CEPAR/pesoenf.pdf \#search=" peso de enfermedad ecuador". Acceso el 14 de marzo de 2010.

39. Lozano R. El peso de la enfermedad en México: avances y desafíos. México, D.F.: Fundación Mexicana para la Salud; 1997.

40. Velásquez Valdivia A. Análisis del estudio de carga de enfermedad en el Perú, MINSA2004, y propuesta metodológica para el ajuste con datos nacionales de morbilidad. Lima: Promoviendo Alianzas y Estrategias; 2006.

41. Ezzati M, López AD, Rodgers A, Vander Hoorn S, Murray CJ. Selected major risk factors and global and regional burden of disease. Lancet. 2002;360(9343):1347-60.

42. República Argentina, Instituto Nacional de Estadísticas y Censos. Censo 2001. Buenos Aires: Instituto Nacional de Estadísticas y Censos; sin año. Hallado en http://www. indec.gov.ar/webcenso/index.asp. Acceso el 14 de marzo de 2010.
43. Rodríguez $\mathrm{T}$, Malvezzi $\mathrm{M}$, Chatenoud $\mathrm{L}$, Bosetti C, Levi F, Negri E, et al. Trends in mortality from coronary heart and cerebrovascular diseases in the Americas: 19702000. Heart. 2006;92(4):453-60.

44. GESICA investigators. Randomised trial of telephone intervention in chronic heart failure: DIAL trial. Br Med J. 2005;331(7514):425

45. Sosa Liprandi MI, González MA, Liprandi AS. Perspectiva de la insuficiencia cardiaca en la Argentina. Medicina. 1999;59(6):787-92.

46. Muratore C, Belziti C, Di Toro D, Gant López J, Mulassi A, Barrios A, et al. Precisión del certificado de defunción comparado con la autopsia verbal: estudio PRISMA. Rev Argent Cardiol. 2006;74(3):211-6

47. Assmann G, Cullen P, Schulte H. Simple scoring scheme for calculating the risk of acute coronary events based on the 10-year followup of the Prospective Cardiovascular Munster (PROCAM) study. Circulation. 2002; 105(3):310-5

48. Jamison D. Investing in health. 2nd ed. New York: Oxford University Press; 2006.

49. Rubinstein A, García Martí S, Souto A, Ferrante D, Augustovski F. Generalized costeffectiveness analysis of a package of interventions to reduce cardiovascular disease in Buenos Aires, Argentina. Cost Eff Resour Alloc. 2009;7:10.

50. Rubinstein A, Belizan M, Discacciati V. Are economic evaluations and health technology assessments increasingly demanded in times of rationing health services? The case of the Argentine financial crisis. Int J Technol Assess Health Care. 2007;23(2):169-76.

Manuscrito recibido el 9 de julio de 2009. Aceptado para publicación, tras revisión, el 7 de marzo de 2010.
ABSTRACT

\section{Estimate of the cardiovascular disease burden attributable to modifiable risk factors in Argentina}

\footnotetext{
Key words

Cardiovascular diseases; cost of illness; potential years of life lost; risk factors;
}

Objective. Estimate the burden of disease, the proportion attributable to the principal modifiable cardiovascular risk factors, and the direct medical cost of hospitalization associated with coronary heart disease and stroke in Argentina.

Methodology. An analitical model was prepared using Argentina's 2005 mortality data and the prevalence of the principal cardiovascular risk factors (hypertension, hypercholesterolemia, overweight, obesity, hyperglycemia, current and past smoking, sedentary lifestyle, and inadequate intake of fruits and vegetables). The burden of disease- -years of potential life lost (YPLL) and years of healthy life lost (YHLL) and hospitalization costs for the cardiovascular diseases analyzed were estimated.

Results. In 2005 over 600000 YHL were lost in Argentina and the number of YPLL due to heart disease and stroke was calculated at 400 000; 71.1\% of the YHLL, $73.9 \%$ of the YPLL, and $76.0 \%$ of the associated costs were attributable to modifiable risk factors. Hypertension was the risk factor with the greatest impact in both men and in women, responsible for $37.3 \%$ of the total cost, 37.5\% of the YPLL, and 36.6\% of the YHLL. Conclusions. Most of the burden of disease from cardiovascular disease in Argentina is associated with modifiable, and therefore preventable, risk factors and could be reduced through population-based and clinical interventions that employ a risk approach; such interventions have already proven to be cost effective, accessible, and feasible in countries like Argentina. Argentina. 